\title{
IS DESVENLAFAXINE EFFECTIVE AND SAFE IN THE TREATMENT OF MENOPAUSAL VASOMOTOR SYMPTOMS? A META-ANALYSIS AND META-REGRESSION OF RANDOMIZED DOUBLE-BLIND CONTROLLED STUDIES
}

\author{
Yifru Berhan', Asres Berhan ${ }^{1}$
}

ABSTRACT

BACKGROUND: During perimenopause, vasomotor symptoms are known to have a detrimental effect on women's functional ability and quality of life. For symptomatic women not eligible for hormonal therapy, desvenlafaxine is an option, but its safety margin and tolerability are not yet determined.

METHODS: A computer-based literature search was done in the databases of MEDLINE, Cochrane library, and HINARI (Health InterNetwork Access to Research Initiative). Meta-analysis was conducted by including double-blind randomized controlled studies on the effectiveness and safety of desvenlafaxine in the treatment of hot flashes. The effectiveness, safety and tolerability of desvenlafaxine were determined by standardized mean differences (SMDs) and Mantel-Haenszel odds ratio. Subgroup analysis based on doses of desvenlafaxine and linear meta-regression analyses were performed for several covariates. Heterogeneity testing, the risk of bias assessment and sensitivity analyses were done.

RESULTS: Desvenlafaxine was associated with a statistically significant reduction in the number and severity of daily moderate to severe hot flashes. The number of nighttime awakenings because of hot flashes was also significantly decreased. However, the rate of desvenlafaxine treatment discontinuation because of adverse events was a significantly higher than placebo treated women and the risk ratios of adverse events like asthenia, hypertension, anorexia, constipation, diarrhea, dry mouth, nausea, dizziness, insomnia, somnolence and mydriasis were very high.

CONCLUSION: Desvenlafaxine is effective in the treatment of hot flashes but it is strongly associated with several adverse events and treatment discontinuation. Further clinical trials focusing only on desvenlafaxine related adverse events are highly warranted before it is approved for public use.

KEY WORDS: clinical trials, desvenlafaxine, hot flash, menopause, meta-analysis

DOI: http://dx.doi.org/10.4314/ejhs.v24i3.4

\section{INTRODUCTION}

Although menopause (the last menses) is a physiological phenomenon that marks the end of a woman's reproductive age, the transition period before and after this signal is usually associated with vasomotor symptoms, vaginal symptoms, urinary incontinence, sexual dysfunction, and troubled sleeping (1). Up to $75 \%$ of the women in the perimenopausal age may experience vasomotor symptoms including hot flushes, sweating, palpitations and feelings of anxiety (26). Vasomotor symptoms are known to have a detrimental effect on women's functional ability and quality of life -poor sleep patterns, frequently disturbed mood and concentration (7-9).

The exact underlying physiological mechanism of vasomotor symptoms is still illusive. Estrogen withdrawal (not low levels of estrogen) is thought to be the most probable expla-

${ }^{1}$ College of Medicine and Health Sciences, Hawassa University, Ethiopia

Corresponding Author: Yifru Berhan, Email: yifrub@yahoo.com 
nation for vasomotor symptoms as evidenced by its absence in gonadal dysgenesis and after some years of menopause, and the exaggerated and rapid onset of these symptoms after bilateral oophorectomy $(10,11)$. The withdrawal of estrogen leads to a reduction in the endorphin and catecholestrogen levels in the hypothalamus, which in turn enhances the release of norepinephrine and serotonin $(12,13)$. Elevated Norepinephrine and serotonin are thought to lower the set point in the thermoregulatory nucleus of the hypothalamus $(14,15)$.

Taking into account the above proposed mechanism, selective serotonin reuptake inhibitors (SSRIs) and serotonin norepinephrine reuptake inhibitors (SNRIs), are under investigation for treatment of vasomotor symptoms (16-21). Furthermore, a meta-analysis of non-hormonal therapies (SSRIs or SNRIs, clonidine, gabapentin, red clover isoflavone extract and soy isoflavone extracts) has demonstrated their effectiveness in the treatment of hot flashes (22). However, the therapeutic effects of these non-hormonal compounds were not as good as estrogen and the primary studies included in that meta-analysis were found to have had methodological deficiencies (22).

Of the non-hormonal compounds, venlafaxine is an antidepressant that acts by inhibiting the reuptake of serotonin and norepinephrine. Its effectiveness in the treatment of menopausal hot flashes appears comparable or superior to other non hormonal compounds like clonidine and gabapentine (23-26). Nevertheless, relative to clonidine, venlafaxine related adverse events were more prominent $(23,24)$.

Desvenlafaxine, the major active metabolite of venlafaxine that is approved for the treatment of major depressive disorder, is also under investigation for treatment of menopausal vasomotor symptoms. Some clinical trials demonstrate its consistent effectiveness in reducing vasomotor symptoms (27-31); while another study doubted its effectiveness (32). The findings of the primary studies on desvenlafaxine effectiveness and safety in the treatment of menopausal vasomotor symptoms were inconclusive. A systematic review of four clinical trials pointed out that the desvenlafaxine treatment on hot flashes has inconsistent effectiveness results (33). Similarly, there were inconsistent results among the clinical trials in the rate of treatment discontinuation.

A meta-analysis of desvenlafaxine efficacy and safety was done some before months by including six studies. However, the meta-analyses were performed with only desvenlafaxine doses of $100 \mathrm{mg}$ and $150 \mathrm{mg}$. Above all, although several statistically significant side effects were identified, a wrong conclusion was made by stating that desvenlafaxine appears safe (34).

Thus, though it was speculated to be the first non-hormonal drug approved by the FDA for vasomotor symptoms treatment (35), its safety and effectiveness is not yet determined. With this background, the primary aim of this meta-analysis was to determine the effectiveness and safety of all assessed doses of desvenlavafaxine $(50 \mathrm{mg}$, $100 \mathrm{mg}, 150 \mathrm{mg}$ and $200 \mathrm{mg}$ ) in the treatment of menopausal vasomotor symptoms by including seven clinical trials.

\section{METHODS}

Search strategy: After coming to an agreement on search terms, electronic based literature search was conducted by two of the authors in the databases of HINARI, Medline and Cochrane library. Via HINARI, the websites of major publishers (Elsevier Science-Science Direct, Nature Publishing Group, Oxford University Press, PsycARTICLES, Science and WileyBlackwell) were searched. Our search was further strengthened by Google scholar search engine and by searching relevant articles from the references' list of retrieved articles. During searching, the following search terms were combined with the Boolean logic (and/or): "desvenlafaxine", "serotonin-norepinephrine reuptake inhibitors" "hot flashes" "hot flushes", "menopause" and "vasomotor symptoms".

Inclusion criteria and study selection: The predefined inclusion criteria were: 1) double-blind randomized controlled studies that weighted the effectiveness and safety of desvenlafaxine against placebo in postmenopausal women who were seeking treatment for hot flashes and/or who experienced at least 7 moderate to severe hot flashes per day; and 2) studies that were published in English and have a duration of therapy not less 
than 12 weeks. The studies included in this work were published between 2005 and February 2013.

Study selection was done by two of the authors in two phases. First, after the titles and abstracts of the retrieved literature were read, they were categorized as "eligible for further screening" and illegible for further screening". Secondly, after screening the whole documents of literature grouped under "eligible for further screening", they were grouped as "eligible for meta-analysis" and "illegible for meta-analysis". When there was disagreement or difference in the grouping of specific articles, it was resolved by discussion and by reviewing the disputing article in detail.

Data extraction: After developing a common data extraction template, the following data were abstracted from the selected studies using standard Excel spreadsheet: name of the first author, year of publication, study design, duration of therapy, dose, sample size, demographics and baseline clinical characteristics, mean change in the daily moderate to severe hot flashes from the baseline and standard deviation (SD) or standard error (SE), mean change in the daily severity score and $\mathrm{SD} / \mathrm{SE}$, mean change in the number of night time awakenings per night, the Greene climacteric scale (GCS) total score, proportion of women with $\geq$ $50 \%$ reduction or $\geq 75 \%$ reduction in the moderate to severe hot flashes, number of women who discontinued the medication due to adverse events and number of women who experienced adverse events.

Operational definitions: In all studies included, women who were taking any hormone therapy or other medications for the treatment of vasomotor symptoms during the clinical trials were excluded. Furthermore, the average daily hot flashes (HFs) severity was calculated as follows: [(number of mild HFs x 1) + (number of moderate HFs x 2) + (number of severe HFs $x$ 3)]/total number of HFs on that day. The GCS total score (36) was determined only by assessing the vasomotor symptoms (hot flashes and night sweats). In this article, vasomotor symptoms and hot flashes are used interchangeably.

Statistical analysis: Since there was moderate variability in the fixed effects model (heterogeneity testing showing 52\% - 58\% variability) of desvenlafaxine effectiveness in the treatment of vasomotor symptoms, the effect size for each study and the pooled effect were computed by the random effects model. Standard mean differences (SMDs) and 95\% confidence intervals $(95 \% \mathrm{CI})$ for the mean change in the number of daily moderate to severe hot flashes from the baselines, mean change in the daily severity score, mean change in the number of nighttime awakenings per night, and the GCS total score were computed using the inverse variance method. Mantel-Haenszel method was used to compute the odds ratios (OR) for hot flashes of greater than or equal to $50 \%$ and $75 \%$ reduction from baseline, and risk ratios (RR) for specific adverse events.

The consistency of the treatment effectiveness across the included studies was assessed by the heterogeneity testing, chi-squared test (Cochrane Q test) and $\mathrm{I}^{2}$ statistics. An $\mathrm{I}^{2}$ value of $\geq 50 \%$ was considered as statistically significant. Subgroup analysis based on doses of desvenlafaxine and linear meta-regression analysis for several covariates or moderators (women's age, baseline BMI, baseline severity of hot flashes, duration from menopause and the proportion of women with natural menopause) were conducted to examine the sources of heterogeneity. Similarly, sensitivity analysis (leaving one study out a time analysis) was done to assess the stability of the pooled values to outliers and the change in heterogeneity. Risk of bias of individual studies was evaluated with the Cochrane risk of bias tool. However, since the studies included in this metaanalysis were fewer than ten, the tests for funnel plot asymmetry were not done as recommended (37). All the statistical analyses were conducted using OpenMeta (analyst) software and comprehensive meta-analysis software.

\section{RESULTS}

As presented in Figure 1 (PRISMA flow chart), through database search, 1,010 articles were identified. Based on proximity of the titles to the objective of this meta-analysis, 70 articles were retrieved. After reviewing the abstracts of the retrieved articles, 15 articles were eligible for full document screening. Finally only 7 articles (27 32,38 ) met the pre-defined inclusion criteria. Four of the studies $(27,28,32,38)$ compared the 
effectiveness and safety of $100 \mathrm{mg} /$ day of desvenlafaxine against placebo; three of the studies $(30,31,38)$ tested two doses of desvenlafaxine $(100 \mathrm{mg} /$ day and $150 \mathrm{mg} /$ day $)$ against placebo; and two studies $(29,38)$ tested four doses of desvenlafaxine $(50 \mathrm{mg} /$ day, 100 $\mathrm{mg} /$ day, $150 \mathrm{mg} /$ day and $200 \mathrm{mg} /$ day) against placebo. The risk of bias assessment did not show bias in randomization, blinding and selective reporting.

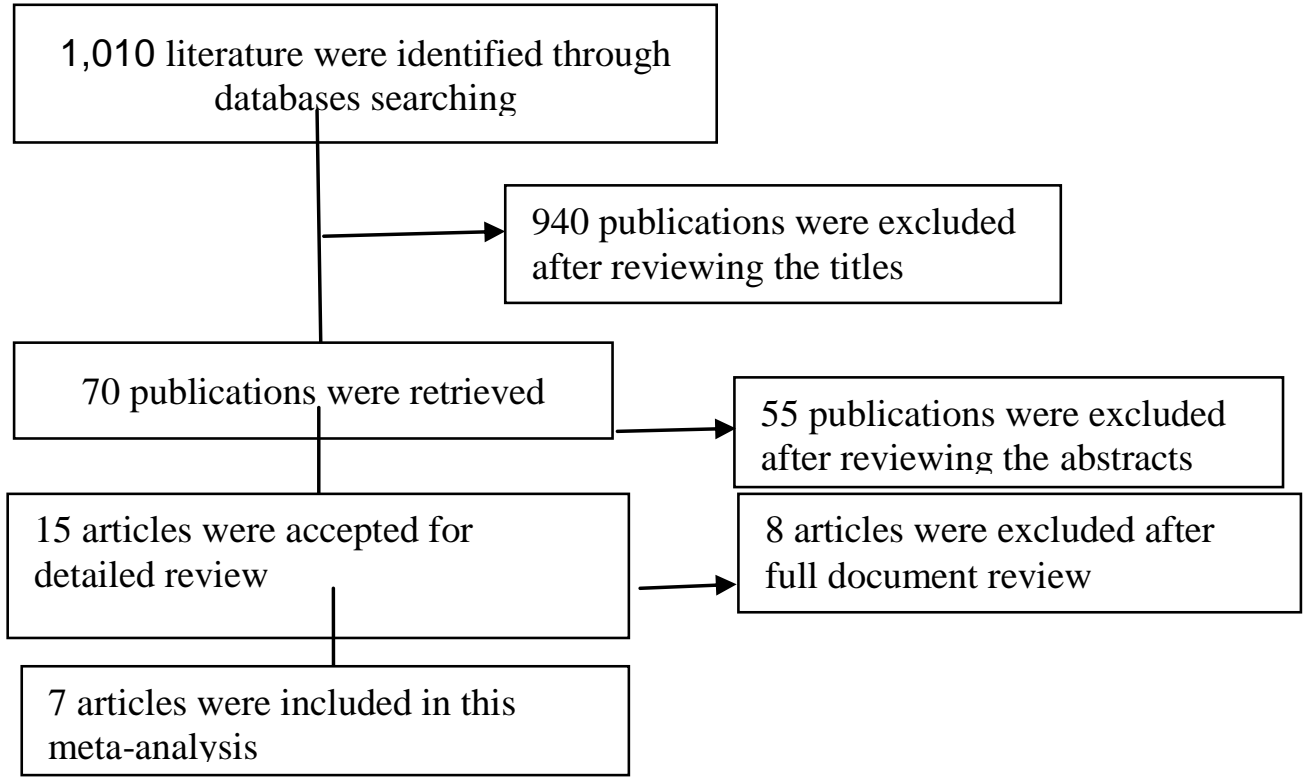

Figure 1: Flow diagram showing studies selection

Overall, in these studies, 2020 women received desvenlafaxine, and 1665 women received placebo. Among desvenlafaxine treated, with moderate inconsistency $\left(\mathrm{I}^{2}=52 \%\right)$, the metaanalysis showed a statistically significant reduction in the number of daily moderate to severe hot flashes from the baseline (over all SMD $=-0.3 ; 95 \%$ CI, -0.41 to -0.22 ) (Figure 2). Except in one study (32), desvenlafaxine $100 \mathrm{mg} /$ day has significantly reduced the recurrence of moderate to severe hot flashes. In other two studies $(29,38)$, although the actual value of the SMD inclines to the left, neither too low $(50 \mathrm{mg})$ nor too high (200 $\mathrm{mg}$ ) dose of desvenlafaxine showed statistically significant reduction in the number of daily moderate to severe hot flashes.

In another analysis, a large number of women in the desvenlafaxine treated group have achieved a reduction in the number of daily moderate to severe hot flashes by $\geq 50 \%$ (overall $\mathrm{OR}=2.5$; $95 \% \mathrm{CI}, 1.84$ to 3.30 ) and $\geq 75 \%$ (overall OR $=$ $2.1 ; 95 \%$ CI, 1.65 to 2.53 ) from the baseline (28, 30-32).
Meta-regression showed that desvenlafaxine treatment outcome was not dependent on women's age, baseline body mass index (BMI), duration after menopause, baseline severity and frequency of hot flashes. Interestingly, desventlafaxine treatment seems more effective in the natural menopause than in the bilateral ophorectomy group (regression coefficient $=-0.01 ; 95 \% \mathrm{CI}$,0.263 to $0.002 ; \mathrm{P}=0.053$ ).

On the other hand, the subgroup analysis based on desvenlafaxine dose $(50 \mathrm{mg} /$ day, 100 $\mathrm{mg} /$ day, $150 \mathrm{mg} /$ day and $200 \mathrm{mg} /$ day) did not demonstrate a significant variation in the mean change of daily moderate to severe hot flashes. The sensitivity analysis, however, verified the stability of the overall SMD (with the withdrawal of any of the studies from the analysis, the overall SMD swings between -0.30 and -0.38). However, when Bouchard et al study (32) was withdrawn from the analysis; there was a significant reduction in the heterogeneity testing ( $\mathrm{I}^{2}$ drops from $52 \%$ to $37 \%$ ). 
Studies/ dose

Archer DF et al study1/ 100mg Archer DF et al study1/ $150 \mathrm{mg}$ Archer DF et al study $2 / 100 \mathrm{mg}$ Archer DF et al study 2 l $150 \mathrm{mg}$ Bouchard P et all $100 \mathrm{mg}$

Pinkerton JV et al study $1 / 100 \mathrm{mg}$ Pinkerton JV et al study $2 / 100 \mathrm{mg}$ Speroff L et al/ $100 \mathrm{mg}$ Speroff $L$ et al/ $150 \mathrm{mg}$ Speroff $L$ et all $200 \mathrm{mg}$ Speroff L et al/ $50 \mathrm{mg}$ Wyrwich et al/ $100 \mathrm{mg}$ Wyrwich ot al/ $150 \mathrm{mg}$ Wyrwich et al/ $200 \mathrm{mg}$ Wyrwich et al/ $50 \mathrm{mg}$

Overall $\left(\mathrm{t}^{\wedge} \mathrm{Z}=52 \%, P=0.010\right)$
SMD (95* CT)
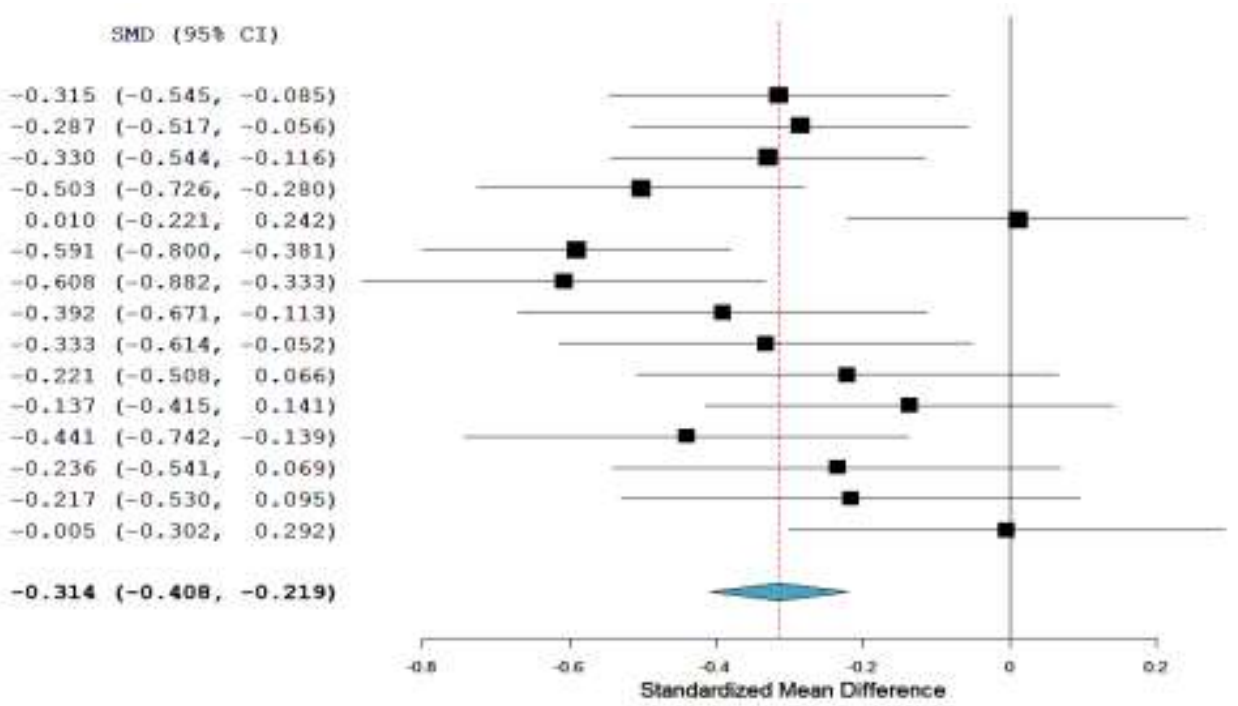

Figure 2: Standardized mean difference of the change in the number of daily moderate to severe hot flashe from the baseline

As shown in figure 3, the meta-analysis of the daily hot flashes severity score has demonstrated a significant reduction in the severity of hot flashes in the desvenlafaxine treated groups (overall SMD $=-0.3 ; 95 \% \mathrm{CI},-0.38$ to -0.17$)$. However, heterogeneity testing still showed the existence of a moderate variability among the included studies $\left(\mathrm{I}^{2}=58 \%\right)$. In the subgroup analysis based on the doses of desvenlafaxine, the significant heterogeneity among the studies was not also explained by the variation in the doses of desvenlafaxine.
Studies/ dose

Archer DF et al study1/ $100 \mathrm{mg}$ Archer DF et al study 1/ $150 \mathrm{mg}$ Archer DF et al studyz/ $100 \mathrm{mg}$ Archer DF et al study2/ $150 \mathrm{mg}$ Bouchard P et al $100 \mathrm{mg}$

Pinkerton JV et al study $1 / 100 \mathrm{mg}$ Pinkerton JV et al study $2 / 100 \mathrm{mg}$ Speroff $L$ et all $100 \mathrm{mg}$ Speroff $L$ et all $150 \mathrm{mg}$ Speroff $L$ et all $200 \mathrm{mg}$ Speroff L et all $50 \mathrm{mg}$ Wyrwich et al/ $100 \mathrm{mg}$

Wyrwich et al/ $150 \mathrm{mg}$

Wyrwich et al/ $200 \mathrm{mg}$ Wyrwich et al/ $50 \mathrm{mg}$

Overall (1^ $2=58 \%, P=0.002)$

SMD (95s cr)
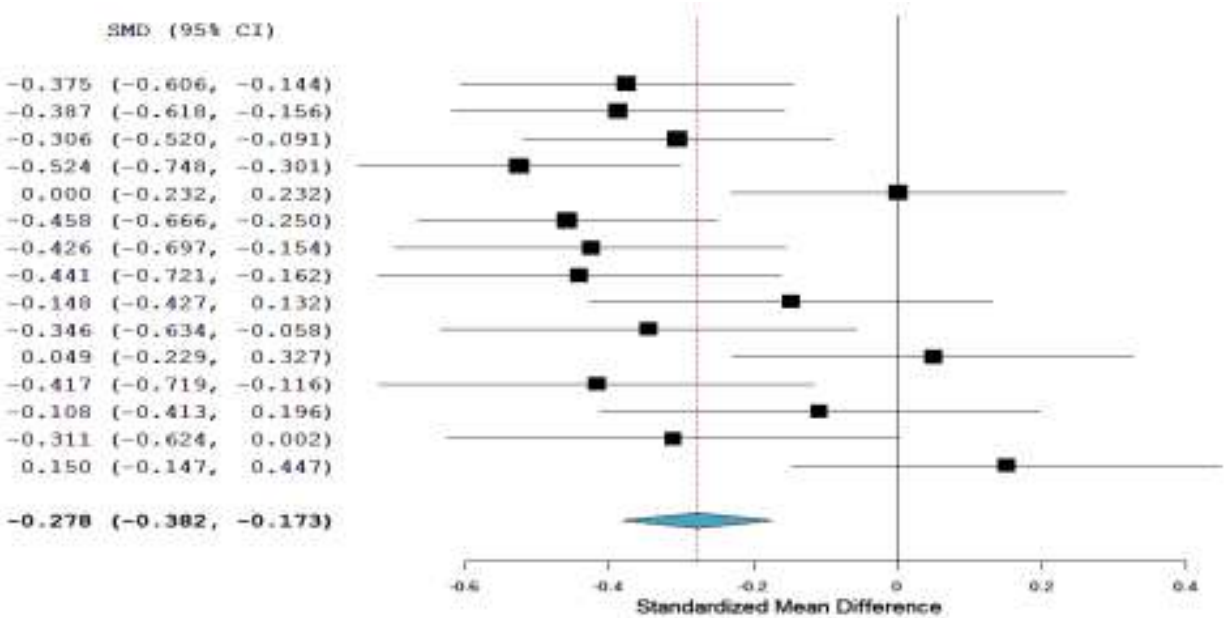

Figure 3: Standardized mean difference of the change in the daily hot flashes severity score (The Greene climacteric scale)

The meta-analysis of awakenings per night because of hot flashes and the change in the GCS, total score have brought additional evidence to support desvenlafaxine effectiveness in the treatment of hot flashes. In other words, the number of awakenings per night because of hot flashes (overall SMD $=-0.3 ; 95 \% \mathrm{CI},-0.38$ to 0.20) (Figure 4) and GCS total score (overall $\mathrm{SMD}=-0.3 ; 95 \% \mathrm{CI},-0.36$ to -0.20$)$ were significantly decreased from baseline in desvenlafaxine treated groups. 

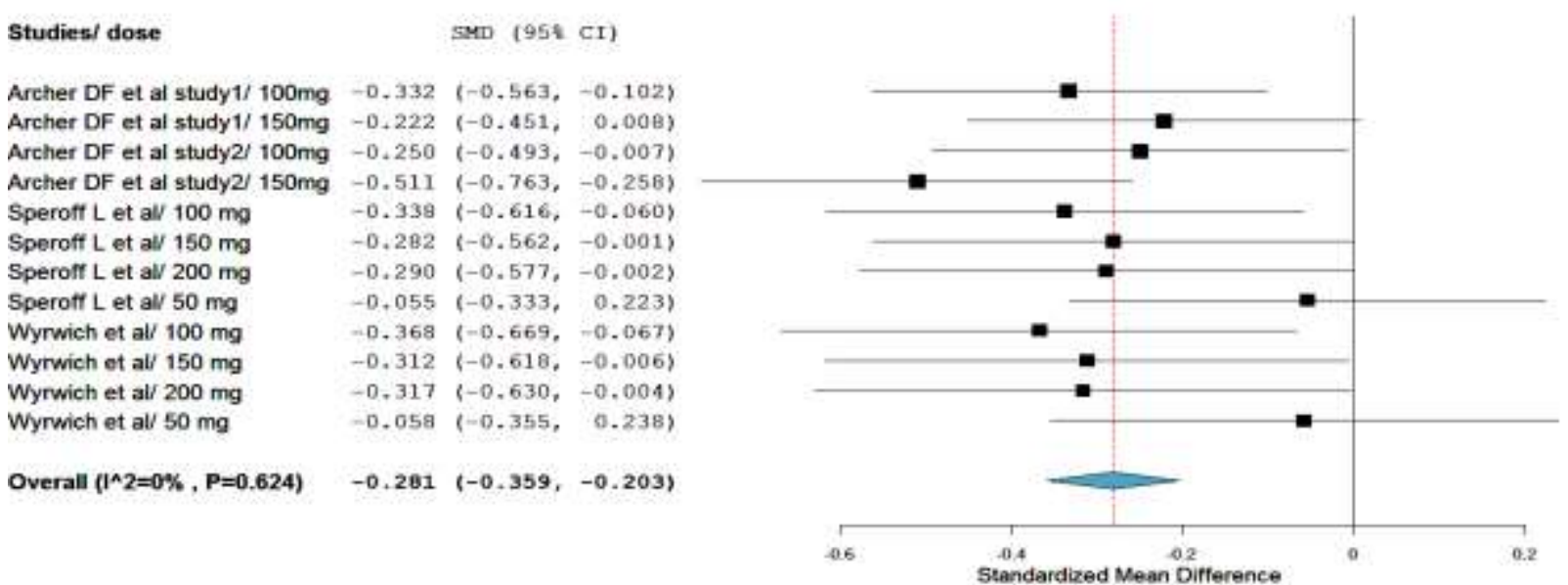

Figure 4: Standardized mean difference of the change in the number of nighttime awakenings due to hot flashes

As shown in figure 5 , the overall rate of desvenlafaxine discontinuation because of adverse events was significantly higher than placebo treated women (overall $\mathrm{OR}=2.4 ; 95 \% \mathrm{CI}, 1.87$ to
3.15). The rate of discontinuation due to desvenlafaxine adverse events was higher in those treated with $150 \mathrm{mg}$ and $200 \mathrm{mg}$ with the exception of one study (31).

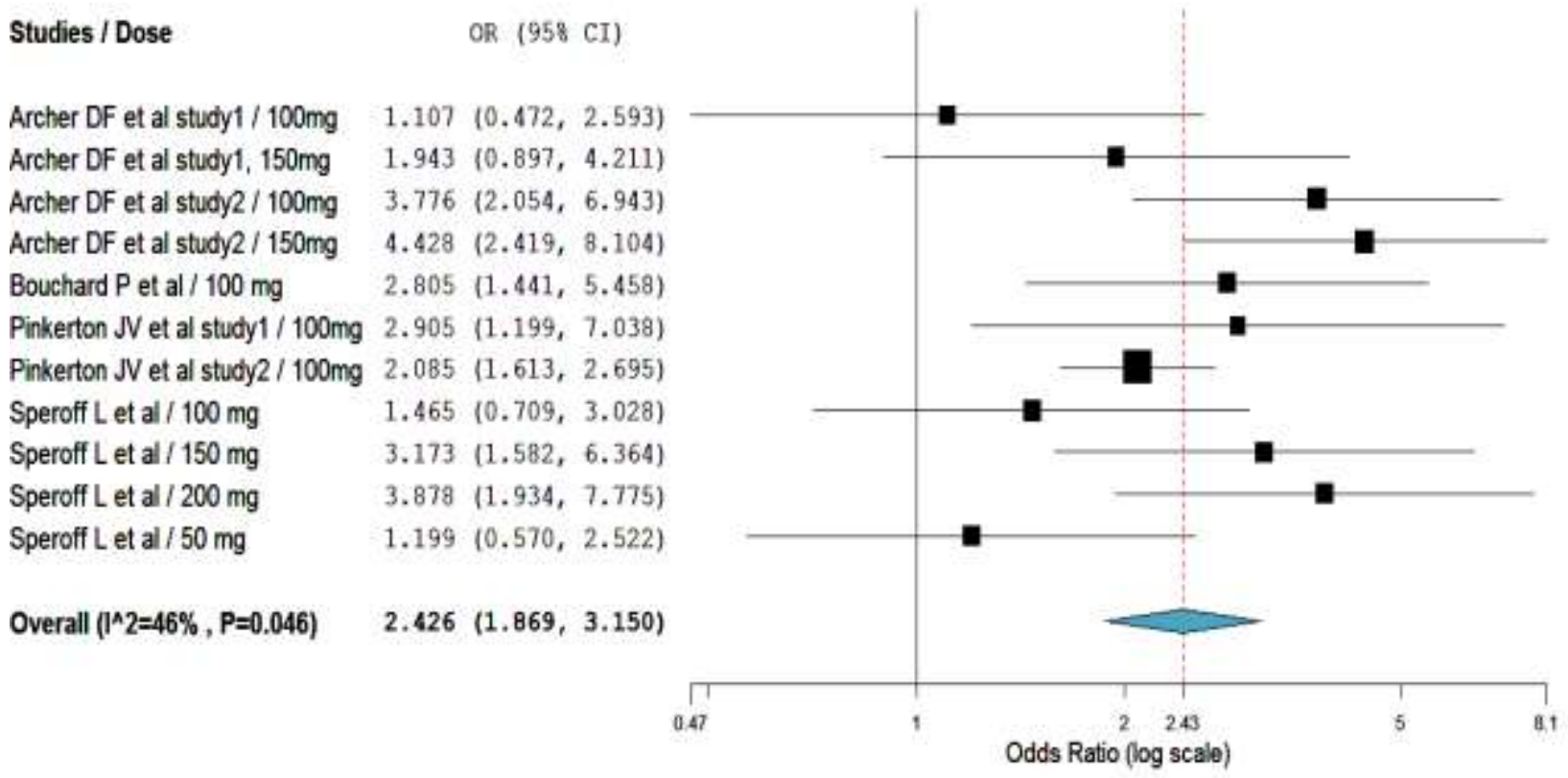

Figure 5: Mantel-Haenszel odds ratio of desvenlafaxine treatment discontinuation due to adverse events

Table 1 summarizes 12 meta-analyses. Hot flashes treated with desvenlafaxine was found to have a highly statistically significant association with several adverse events like asthenia $(\mathrm{OR}=2.3)$, hypertension $(\mathrm{OR}=2.2)$, anorexia $(\mathrm{OR}=3.6)$, constipation $(\mathrm{OR}=2.5)$, diarrhea $(\mathrm{OR}=1.6)$, dry mouth $(\mathrm{OR}=5.9)$, nausea $(\mathrm{OR}=4.3)$, dizziness
$(\mathrm{OR}=2.4)$, insomnia $(\mathrm{OR}=1.9)$, somnolence $(\mathrm{OR}$ $=4.8)$ and mydriasis $(\mathrm{OR}=7.5)$ (Table1). As evidenced by their high risk ratios, the majorities of adverse events were encountered among women who were treated with high doses of desvenlafaxine (150mg and 200mg). 
Table 1: Summary of the Mantel-Haenszel risk ratio of desvenlafaxine treatment adverse events

\begin{tabular}{llcc}
\hline Adverse events & Risk Ratio (M-H, 95\% CI) & $\mathbf{I}^{\mathbf{2}}$ & $\begin{array}{c}\text { Number of participants } \\
\text { (Desvenlafaxine/placebo) }\end{array}$ \\
\hline Any adverse events & $1.15[1.08-1.22]^{* *}$ & $56 \%$ & $1271 / 952$ \\
Asthenia & $2.25[1.67-3.03]^{* * *}$ & $12 \%$ & $1432 / 1122$ \\
Hypertension & $2.16[1.30-3.61]^{* *}$ & $0 \%$ & $1274 / 970$ \\
Anorexia & $3.56[2.09-6.06]^{* * *}$ & $0 \%$ & $1274 / 970$ \\
Constipation & $2.51[1.79-3.53]^{* * *}$ & $49 \%$ & $2698 / 2364$ \\
Diarrhea & $1.57[1.10-2.23]^{*}$ & $10 \%$ & $1474 / 1160$ \\
Dry mouth & $5.87[4.34-7.95]^{* * *}$ & $0 \%$ & $2698 / 2364$ \\
Nausea & $4.31[3.40-5.46]^{* * *}$ & $40 \%$ & $2698 / 2364$ \\
Dizziness & $2.39[1.97-2.91]^{* * *}$ & $0 \%$ & $2498 / 2174$ \\
Insomnia & $1.94[1.54-2.44]^{* * *}$ & $0 \%$ & $1432 / 1122$ \\
Somnolence & $4.81[3.41-6.79]^{* * *}$ & $0 \%$ & $2698 / 2364$ \\
Mydriasis & $7.52[2.66-21.24]^{* *}$ & $0 \%$ & $1274 / 970$ \\
\hline
\end{tabular}

$* \mathrm{P}<=0.01 ; * * \mathrm{P}<0.0001 ; * * * \mathrm{P}<0.00001$

\section{DISCUSSION}

In support of previous meta-analyses done on other non-hormonal compounds $(22,39)$, this meta-analysis has demonstrated a significant reduction of recurrence and severity of menopausal hot flashes in women who were treated with desvenlafaxine. Furthermore, a significant number of women who were treated with desvenlafaxine achieved a greater than or equal to $75 \%$ reduction in the number of daily moderate to severe hot flashes. Among women who were treated with desvenlafaxine, the number of nighttime awakenings per night due to hot flashes also dropped significantly. However, a significant number of women who were treated with desvenlafaxine experienced more adverse events and discontinuation of the drug than placebo treated women.

The high statistically significant association of desvenlafaxine with all selected adverse events (Risk ratio as high as 7.5), which was complemented by more than 2.4-fold increased odds of treatment discontinuation due to adverse events, makes the safety and tolerability of this drug for the treatment of perimenopausal vasomotor symptoms questionable. However, it should be noted that the majority of the women who discontinued the desvenlafaxine treatment were put on relatively high dose, which probably indicates the dose related adverse events as stated in Speroff et al clinical trial (29). Furthermore, the finding of higher risk ratio of adverse events among women treated with high dose desvenlafaxine is also another evidence to question the safety margin of desvenlafaxine. Dose titration and tapering may be options to alleviate the observed adverse events and treatment discontinuation as reported in one of the clinical trials (31) and recommended by reviewers (33). Literature reviews have also shown that adverse events are the major problems of several other non-hormonal agents used for the treatment of hot flashes $(40,41)$.

However, in a study focusing only on adverse events, it was reported that menopausal vasomotor symptoms treated with desvenlafaxine $100 \mathrm{mg} /$ day compared with placebo did not show an increased risk of cardiovascular (including hypertension), cerebrovascular, or hepatic events (40). This is probably because of the fact that the studies included in this meta-analysis were not primarily designed to address the side effects of desvenlafaxine, which could be taken as a limitation to draw a conclusion. As recommended in a literature (42), since the sample sizes of the individual studies included in a meta-analysis did not provide adequate power to test rare adverse events, a separate research design has to be developed primarily intended to assess the adverse events. This is also one of the recommendations of this meta-analysis before declaring the safety and tolerability of desvenlafaxine. 
Until proved otherwise, however, desvenlafaxine $100 \mathrm{mg} /$ day seems effective and relatively safe in the treatment of vasomotor symptoms. The desvenlafaxine $50 \mathrm{mg} /$ day dose was not effective in all assessed outcome indicators (change in nighttime awakenings, frequency and severity of hot flashes), but it was not strongly associated with treatment discontinuation and adverse events probably because it was well tolerated. The desvenlafaxine $150 \mathrm{mg} /$ day and $200 \mathrm{mg} /$ day doses were not also consistently effective, but were strongly associated with treatment discontinuation and adverse events in the majority of the clinical trials. Furthermore, there was no significant difference in the proportion of hot flashes among women treated with $100 \mathrm{mg}, 150 \mathrm{mg}$ and 200mg; the symptom reduction was in the range of $60 \%$ $65 \%$ (29-31). Therefore, the authors would like to recommend clinical trials focusing only on adverse events of desvenlafaxine with a dose of $100 \mathrm{mg} /$ day.

Meta-regression has shown that desvenlafaxine treatment seems more effective in women with the natural than in the surgical menopause. However, this finding should be interpreted very cautiously. Firstly, the number of women with surgical menopause was less than one-third of the women with the natural menopause, which may not be representative of the general population. Secondly, the course of estrogen withdrawal (attributed for vasomotor symptoms) in the natural menopause is a slow process, while in the surgical case, it is an abrupt onset. Surgical menopause is known to have fast onset and severe vasomotor symptoms $(33,43,44)$ that may not respond to desvenlafaxine treatment. Thirdly, although the meta-regression did not demonstrate significant variation, it is known that the longer the duration of postmenopause, the lesser the severity of vasomotor symptoms, which is likely to happen more in the natural than in the surgical menopause.

In general, so far, the most effective treatment that is approved by the Food and Drug Administration of America (FDA) for vasomotor symptoms is hormone replacement therapy. Nevertheless, there are several absolute (unexplained vaginal bleeding, thromboembolic disease, breast cancer, severe liver disease, coronary artery disease, type I endometrial cancer) and relative (migraine headache, ovarian cancer, leiomyoma, and gall bladder disease) contraindications for hormonal therapy $(45,46)$. Specific to breast cancer, since antiestrogenic medications and ophorectomy are part of the treatment modalities, vasomotor symptoms are more common and severe $(43,44)$. As a result, desvenlafaxine can have several indications to treat vasomotor symptoms provided that the safety margin and patients' compliance are re-evaluated. This study has several limitations. Among the studies included in it, the heterogeneity testing showed moderate variability. Although the subgroup analysis and meta-regression did not support our assumptions, several factors are attributed to the observed heterogeneity: the desvenlafaxine dose variation, baseline differences in severity and frequency of hot flashes, women's age and BMI, ethnicity, duration since menopause, and type of menopause (surgical or natural). Furthermore, previous hormonal therapies and adjuvant traditional therapies during the clinical trials were not reported in the selected studies. The duration of therapy in the clinical trials was also limited to 12 weeks. What is more, we are not sure that we have missed articles written in nonEnglish languages.

In conclusion, this meta-analysis has shown that desvenlafaxine was associated with a statistically significant reduction in the number and severity of daily moderate to severe hot flashes from the baseline. Similarly, the number of nighttime awakenings because of hot flashes was significantly decreased. Desventlafaxine treatment seems more effective in women with the natural than with the surgical menopause. However, the rate of desvenlafaxine discontinuation because of adverse events was a significantly higher than placebo treated women and the risk ratios of adverse events like asthenia, hypertension, anorexia, constipation, diarrhea, dry mouth, nausea, dizziness, insomnia, somnolence and mydriasis were very high. In the treatment of hot flashes, further clinical trials on desvenlafaxine related adverse events are highly warranted before it is approved for public use. 


\section{REFERENCES}

1. Pachman DR, Jones JM and Loprinzi CL. Management of menopause-associated vasomotor symptoms: Current treatment options, challenges and future directions. International $J$ Women's Health 2010:2 123-135.

2. Umland EM. Treatment strategies for reducing the burden of menopause-associated vasomotor symptoms. J Manag Care Pharm 2008; $14(2$ (suppl S)):S14-S19.

3. Freeman EW, Sherif K. Prevalence of hot flushes and night sweats around the world: a systematic review. Climacteric 2007; 10:197214.

4. McKinlay SM, Jefferys M. The menopausal syndrome. Br J Prev Soc Med 1974;28:108-15.

5. Greendale GA, Lee NP, Arriola ER. The menopause. Lancet 1999; 353:571-80.

6. Finck G, Barton DL, Loprinzi CL, et al. Definitions of hot flashes in breast cancer survivors. J Pain Symptom Manage 1998; 16:327-33.

7. Utian WH. Psychosocial and socioeconomic burden of vasomotor symptoms in menopause: A comprehensive review. Health and Quality of Life Outcomes 2005; 3:47. Available from: URL:http://www.hqlo.com/content/3/1/47.

8. Carpenter JS. The hot flash related daily interference scale: A tool for assessing the impact of hot flashes on quality of life following breast cancer. J Pain Symptom Manage 2001; 2:979-89.

9. Daly E, Gray A, Barlow D, McPherson K, Roche M, Vessey M. Measuring the impact of menopausal symptoms on quality of life. BMJ 1993; 307(6908):836-840.

10. Sturdee DW. The menopausal hot flush anything new? Maturitas 2008; 60(1):42-49.

11. Casper RF, Yen SS, Wilkes MM. Menopausal flushes: A neuroendocrine link with pulsatile luteinizing hormone secretion. Science 1979; 205:823-5.

12. Shanafelt TD, Barton DL, Adjei aa, Loprinzi CL. Pathophysiology and treatment of hot flashes. Mayo Clin Proc 2002; 77:1207-18.

13. Archer DF, Sturdee DW, Baber R, et al. Menopausal hot flushes and night sweats: where are we now? Climacteric 2011; 14(5):515-528.

14. Berendsen HH. The role of serotonin in hot flushes. Maturitas 2000; 36(3):155-164.

15. Freedman RR. Physiology of hot flashes. Am J Hum Biol 2001; 13(4):453-464.
16. Stearns V, Slack R, Greep N, et al. Paroxetine is an effective treatment for hot flashes: results from a prospective randomized clinical trial. $J$ Clin Oncol 2005; 23(28):6919-6930.

17. Stearns V, Beebe KL, Iyengar M, Dube E. Paroxetine controlled release in the treatment of menopausal hot flashes: a randomized controlled trial. JAMA 2003; 289(12):2827-2834.

18. Butt DA, Lock M, Debra A. Butt LJE, Ross S, Moineddine R. Gabapentin for the treatment of menopausal hot flashes: a randomized controlled trial. Menopause: The Journal of The North American Menopause Society 2008; 13(2):310 318.

19. Aguirre W, Chedraui P, Mendoza J, Ruilova I. Gabapentin vs. low-dose transdermal estradiol for treating post-menopausal women with moderate to very severe hot flushes. Gynecological Endocrinology 2010; 26(5):333337.

20. Loibl S, Schwedler K, Minckwitz G von, Strohmeier R, Mehta KM, Kaufmann M. Venlafaxine is superior to clonidine as treatment of hot flashes in breast cancer patients - a double-blind, randomized study. Annals of Oncology 2007; 18:689-693.

21. Buijs C, Mom CH, Willemse PHB, et al. Venlafaxine versus clonidine for the treatment of hot flashes in breast cancer patients: a doubleblind, randomized cross-over study. Breast Cancer Res Treat 2009; 115:573-580.

22. Nelson HD, Vesco KK, Haney E, et al. Nonhormonal therapies for menopausal hot flashes systematic review and meta-analysis. JAMA 2006; 295(17):2057-2071.

23. Buijs C, Mom $\mathrm{CH}$, Willemse PHB, et al. Venlafaxine versus clonidine for the treatment of hot flashes in breast cancer patients: a doubleblind, randomized cross-over study. Breast Cancer Res Treat 2006; 115:573-580.

24. Boekhout AH, Vincent AD, Dalesio OB, et al. Management of hot flashes in patients who have breast cancer with venlafaxine and clonidine: a randomized, double-blind, placebo-controlled trial. J Clin Oncol 2011; 29(29):3862-8.

25. Loibl S, Schwedler K, Minckwitz G von, Strohmeier R, Mehta KM, Kaufmann M. Venlafaxine is superior to clonidine as treatment of hot flashes breast cancer patients - a doubleblind, randomized study in. Annals of Oncology 2007; 18:689-693.

26. Bordeleau L, Pritchard KI, Loprinzi CL, et al. Multicenter, Randomized, Cross-Over Clinical 
Trial of Venlafaxine Versus Gabapentin for the Management of Hot Flashes in Breast Cancer Survivors. J Clin Oncol 2010; 28(35):51475152.

27. Pinkerton JV, Archer DF, Guico-Pabia CJ, Hwang E, Cheng RJ. Maintenance of the effectiveness of desvenlafaxine in menopausal vasomotor symptoms: a 1-year randomized controlled trial. Menopause: The Journal of The North American Menopause Society 2013; 20(1):38-46. (Study 2).

28. Pinkerton JV, Constantine G, Hwang E, Cheng RJ. Desvenlafaxine compared with placebo for treatment of menopausal vasomotor symptoms: a 12-week, multicenter, parallel-group, randomized, double-blind, placebo-controlled effectiveness trial. Menopause: The Journal of The North American Menopause Society 2013; 20(1):28-37. (Study 1).

29. Speroff L, Gass M, Constantine G, Olivier S. Effectiveness and Tolerability of Desvenlafaxine Succinate Treatment for Menopausal Vasomotor Symptoms A Randomized Controlled Trial. Obstetrics \& Gynecology 2008; 111(1):77-87.

30. Archer DF, Dupont CM, Constantine GD, Pickar JH, Olivier S. Desvenlafaxine for the treatment of vasomotor symptoms associated with menopause: a double-blind, randomized, placebo-controlled trial of effectiveness and safety. Am J Obstet Gynecol 2009; 200:238.e231-238.e210. (Study 2).

31. Archer DF, Seidman L, Constantine GD, Pickar $\mathrm{JH}$, Olivier S. A double-blind, randomly assigned, placebo-controlled study of desvenlafaxine effectiveness and safety for the treatment of vasomotor symptoms associated with menopause. Am J Obstet Gynecol 2009; 200:172.e1-172.e10. (Study 1).

32. Bouchard P, Panay N, de Villiers TJ, et al. Randomized placebo- and active-controlled study of desvenlafaxine for menopausal vasomotor symptoms. Climacteric 2012; 15:1220.

33. Umland EM, Falconieri L. Treatment options for vasomotor symptoms in menopause: focus on desvenlafaxineInternational Journal of Women's Health 2012; 4: 305-319.

34. Sun Z, Hao Y, Zhang M. Efficacy and safety of desvenlafaxine treatment for hot flashes associated with menopause: a meta-analysis of randomized controlled trials. Gynecologic and Obstetric Investigation 2013; 75(4): 255-262.
35. Shifren JL. Management of Hot Flashes: Alternatives to Estrogen Therapy. Menopause management 2009; 18(2):28-32.

36. Greene JG. Constructing a standard climacteric scale. Maturitas 1998;29:25-31

37. Sterne JAC, Sutton AJ, Ioannidis JPA, et al Recommendations for examining and interpreting funnel plot asymmetry in metaanalyses of randomised controlled trials. BMJ2011, 342:d4002. doi:10.1136/bmj.d4002.

38. Toulis KA, Tzellos T, Kouvelas D, Goulis DG. Gabapentin for the treatment of hot flashes in women with natural or tamoxifen-induced menopause: A systematic review and metaanalysis. Clinical Therapeutics 2009; 31(2):22135.

39. Wyrwich KW, Spratt DI, Gass M, Yu H, Bobula JD. Identifying meaningful differences in vasomotor symptoms among menopausal women. Menopause 2008; 15(4): 698 -705.

40. Archer DF, Pinkerton JV, Guico-Pabia CJ, Hwang E, Cheng RJ. Cardiovascular, cerebrovascular, and hepatic safety of desvenlafaxine for 1 year in women with vasomotor symptoms associated with menopause. Menopause 2012; 20(1): 47-56.

41. Stearns V and Loprinzi CL. New Therapeutic Approaches for Hot Flashes in Women. $J$ Support Oncol 2003; 1:11-21.

42. Walker E, Hernandez AV and Kattan MW. Meta-analysis: Its strengths and limitations. Clev Clin J med 2008; 75 (6): 431-439.

43. Carpenter JS, Andrykowski MA, Cordova M, et al. Hot flashes in postmenopausal women treated for breast carcinoma: prevalence, severity, correlates, management, and relation to quality of life. Cancer 1998; 82(9):1682-91.

44. Couzi RJ, Helzlsouer KJ, Fetting JH. Prevalence of menopausal symptoms among women with a history of breast cancer and attitudes toward estrogen replacement therapy. J Clin Oncol 1995; 13(11):2737-44.

45. Nelson HD. Postmenopausal estrogen for treatment of hot flashes: clinical applications. JAMA 2004; 291(13):1621-1625.

46. WIKIPEDIA. The free encyclopedia. Hormone replacement therapy (Menopause). http://en.wikipedia.org/wiki/HRT (menopause) 